\title{
Analysis of the anti-tumor effect of cetuximab using protein kinetics and mouse xenograft models
}

Teppei Matsuo, Satoshi S Nishizuka*, Kazushige Ishida, Takeshi Iwaya, Miyuki Ikeda and Go Wakabayashi

\begin{abstract}
Background: The binding of EGFR and its ligands leads to autophosphorylation of receptor tyrosine kinase as well as subsequent activation of signal transduction pathways that are involved in regulating cellular proliferation, differentiation, and survival. An EGFR inhibitor, cetuximab binds to EGFR and consequently blocks a variety of cellular processes. KRAS/BRAF mutations are known to be associated with a low response rate to cetuximab. In the present study, to clarify the anti-tumor mechanisms of cetuximab, we evaluated the KRAS/BRAF status, phosphorylation level of the EGFR pathway, and the tumor suppression effect in vivo, using a human colon cancer cell line HT29, which exhibited the highest EGFR expression in response to the cetuximab therapy among the 6 colorectal cancer cell lines tested.

Findings: The conventional growth suppression assay did not work efficiently with cetuximab. EGF, TGF- $\alpha$, and IGF activated the EGFR/MAPK cell signaling pathway by initiating the phosphorylation of EGFR. Cetuximab partially inhibited the EGFR/MAPK pathway induced by EGF, TGF- $\alpha$, and IGF. However, cetuximab exposure induced the EGFR, MEK, and ERK1/2 phosphorylation by itself. Mouse xenograft tumor growth was significantly inhibited by cetuximab and both cetuximab-treated and -untreated xenograft specimens exhibited phosphorylations of the EGFR pathway proteins.

Conclusions: We have confirmed that cetuximab inhibited the EGFR/MAPK pathway and reduced tumor growth in the xenografts while the remaining tumor showed EGFR pathway activation. These results suggest that: ( $i$ ) The effect of cetuximab in growth signaling is not sufficient to induce complete growth suppression in vitro; ( ii ) timecourse monitoring may be necessary to evaluate the effect of cetuximab because EGFR signaling is transmitted in a minute order; and ( iii ) cetuximab treatment may have cells acquired resistant selectively survived in the heterogeneous cancer population.
\end{abstract}

\section{Background}

The epidermal growth factor receptor (EGFR) is a transmembrane glycoprotein that constitutes one of four members of the erbB family of tyrosine kinase receptors [1]. EGFR regulates the key processes of cell biology, including proliferation, survival, and differentiation during development and tissue homeostasis [2]. One of the most common approaches to inhibit EGFR signaling in the anticancer therapeutic context has been to develop monoclonal antibodies against EGFR. The monoclonal

\footnotetext{
* Correspondence: snishizu@iwate-med.ac.jp

Molecular Therapeutics Laboratory, Department of Surgery, Iwate Medical University School of Medicine, 19-1 Uchimaru, Morioka 020-8505, Iwate Japan
}

(c) 2011 Nishizuka et al; licensee BioMed Central Ltd. This is an open access article distributed under the terms of the Creative Commons Attribution License (http://creativecommons.org/licenses/by/2.0), which permits unrestricted use, distribution, and reproduction in any medium, provided the original work is properly cited. antibody 225 (i.e. cetuximab) is one of several antibodies raised by inoculation of mice with A431 epidermoid carcinoma cells [3] and it was subsequently selected to generate a human mouse chimeric molecule for clinical development [4]. Cetuximab (marketed under the name Erbitux) is a chimeric (mouse/human) monoclonal antibody that inhibits the epidermal growth factor receptor (EGFR) [3]. It has been given by intravenous infusion to treat metastatic colorectal cancer and head and neck cancer [5].

It has been reported that cetuximab increases survival in patients with advanced CRC when administered in combination with irinotecan and fluorouracil [6]. Results from other clinical trials have also indicated that the 
benefit from the addition of cetuximab to first-line chemotherapy seems to be restricted to patients with the wild-type KRAS gene; with the best outcomes being observed in those with unmutated forms of both the KRAS and BRAF genes [6-8]. A detailed understanding of the mechanisms controlling cetuximab antitumor activity is necessary to optimize its therapeutic efficacy and to identify those patients who are likely to benefit from the treatment. Although KRAS and BRAF gene status has been considered as a meaningful biomarker, EGFR signaling can also be regulated by several ligands, receptors and cross-talk with other pathways. Hence, these gene mutations may contribute to individual tumors in varying degrees. Moreover, EGFR signaling could change in minutes order so it is crucial to monitor the dynamics of signal transduction to understand the intrinsic biological entity of tumor growth, in addition to the representative gene mutation status. To understand such signaling kinetics and tumor growth suppression in response to cetuximab administration, we used a colorectal cancer cell line, HT29, which expressed the highest EGFR level of 6 colorectal cancer cell lines tested. Here, we report the signaling alteration of the EGFR/MAPK pathway initiated by three types of growth factors in the presence/absence of cetuximab in vitro. The protein phosphorylation status of the xenograft tumor treated with cetuximab will also be discussed.

\section{Material and methods Colon cancer cell lines}

Six human colorectal cancer (CRC) cell lines were used for molecular characterization. HT29 was obtained from the American Type Culture Collection. HCT116 was obtained from the U.S. National Cancer Institute and CW2, JHSK-rec, JHCOLO-YI, and TT1TKB were obtained from the RIKEN Cell Bank. HT29, CW2, and HCT116 were grown in RPMI 1640 supplemented with $10 \%$ fetal bovine serum. JHCOLO-YI and JHSK-rec were grown in DMEM/F12 with $10 \%$ FBS and $0.1 \mathrm{mM}$ non-essential amino acid and TT1TKB was grown in DMEM with $10 \%$ FBS. All cell lines were incubated at $37^{\circ} \mathrm{C}$ with $5 \% \mathrm{CO}_{2}$.

\section{Reagents}

The following 3 growth factors were used for growth signal stimulation: Epidermal Growth Factor (EGF, SIGMA-ALDRICH), Transforming Growth Factor- $\alpha$ (TGF- $\alpha$, Pepro Tech House), and Insulin-like growth factor-I human (IGF, SIGMA-ALDRICH). Each growth factor was dissolved in the medium at the following concentrations: EGF, 100ng/ml; TGF- $\alpha, 50 \mathrm{ng} / \mathrm{ml}$; and IGF, 50ng/ml. Fluorouracil (5-FU, Kyowa Hakko Kirin), an anti-human EGFR monoclonal antibody, cetuximab
(Erbitax, Bristol-Myers K.K.) were used in a serial dilution for growth suppression assays in vitro. When cetuximab was injected into nude mice (described details below), the dose was $10 \mathrm{mg} / \mathrm{kg}$.

\section{DNA sequencing}

Human KRAS exon 2 (codon 12,13) and BRAF sequencing were performed with genomic DNA subjected to PCR amplification with the following set of intronic primers: KRAS forward 5'-GGCCTGCTGAAAATGACTGA-3', reverse 5'-GTCCTGCACCAGTAATA TGC-3' [9]; and BRAF exon 15 (codons 582-620) forward 5'-TGTTTTCCTTTACTTACTACACCTCA-3', reverse 5'-TCAGTGGAAAAATAGCCTCAA-3' [10]. The PCR products were then sequenced using the Big Dye Terminator V3.1 (Applied Biosystems) according to the protocol supplied by the manufacturer.

\section{Growth suppression assay}

Cells (5,000 for cell/swell) were seeded on to a 96-well plate in an appropriate medium with FBS. Forty-eight hours later, the cells were treated with cetuximab with a 10 -fold series of concentrations, and incubated for another 48 hours. Water-soluble tetrazolium salts were added to each well and incubated for 3-6 hours at $37^{\circ} \mathrm{C}$ (CCK-8, Dojindo). Absorbance was measured at 450 $\mathrm{nm}$, with a reference filter at 520-540 $\mathrm{nm}$, using a microplate reader.

\section{Western blot}

Cells were seeded in a T-25 cell culture flask in RPMI1640 containing 10\% FBS. After the cells reached $80 \%$ confluence, the medium was replaced with serumfree RPMI for 24 hours. Effects by serum starvation, such as induction of apoptosis, have been reported for various types of cells including gliomas, bladder and colon carcinomas [11-14]. There are several reports describing the effects of serum starvation in HT29 cells for 24 hours $[15,16]$ and this procedure has been well accepted as a way of seeing the effect of cell growth signaling in general [17]. In the present study, we decided to set a 24 hours serum starvation sample as the control for the following EGFR signaling study. After the serum starvation was completed, the cells were stimulated with combinations of EGF $100 \mathrm{ng} / \mathrm{ml}$, TGF $-\alpha 50 \mathrm{ng} / \mathrm{ml}$, and IGF 50ng/ml, and incubated with cetuximab $300 \mu \mathrm{g} / \mathrm{ml}$, resulting in a total of 7 conditions for $0,1,5,10,15$ minutes. The cells were then washed with PBS and lysed with Pink Buffer containing $9 \mathrm{M}$ urea (Sigma), 4\% 3-[(3-cholamidopropyl)dimethylammonio]-1-propanesulfonate (CHAPS; Calbiochem), 2\% pH 8.0-10.5 Pharmalyte (Amersham Pharmacia Biotech, Piscataway, NJ), and 65 mM DTT (Amersham Pharmacia Biotech) [18]. The full fraction of protein was resolved using NuPAGE ${ }^{\circledR} 4$ - 
$12 \%$ Bis-Tris Gel electrophoresis under reducing conditions and transferred to a nitrocellulose membrane. The resulting membranes were blocked with 5\% nonfat dried milk (cell signaling technology) in $0.1 \%$ Tween 20 (Bio$\mathrm{Rad}$ ) in PBS for 1 hour. Membranes were then incubated with primary antibodies; the dilution factors were all 1:1000 EGFR and p-EGFR(Tyr1045) (Cell Signaling Technology), p-MEK(ser218/ser222) (ECM Biosciences), p-ERK(Thr-202/Tyr-204) and pan-Actin (Thermo), overnight at $4^{\circ} \mathrm{C}$. Next, the membranes were washed 3 times for 5 minutes in $0.1 \%$ Tween 20 in PBS, incubated with an HRP-conjugated secondary antibody for 1 hour at $4^{\circ} \mathrm{C}$, and washed 3 times for 5 minutes in $0.1 \%$ Tween 20 in PBS. Chemiluminescence detection reagents (Thermo) were incubated with the membranes for 10 minutes, which was followed by film development. The signal intensities of phosphorylated EGFR, MEK, and ERK protein levels are expressed in an arbitrary unit (AU).

\section{Xenograft model}

HT29 cells $\left(1.0 \times 10^{7}\right)$ were injected subcutaneously into the right and left side of the back of 5 week old female athymic nude mice (CLEA japan: Central laboratory for experimental animals Japan). Tumor volume was calculated every 2-3 days with a caliper and using the following formula: Volume $=$ length $\times$ width $\times$ height. After the tumors reached approximately $100 \mathrm{~mm}^{3}$, either $40 \mu \mathrm{l}$ of saline solution or $40 \mu \mathrm{l}$ of cetuximab solution $(5 \mathrm{mg} /$ $\mathrm{ml}$ ) was intravenously administered to each mouse twice a week. Animals care was in accordance with our institution's guidelines. All animal experiments in this study were approved by the Iwate Medical University Ethical Committee for Animal Experiment Regulation, 22-007, Morioka, Japan.

\section{Immunohistochemistry}

After euthanization of the mice, the tumors were excised and preserved in $10 \%$ formalin. Hematoxylin and eosin (HE) staining was performed using a standard technique. Immunohistochemical (IHC) staining procedures for total EGFR were as follows: Paraffin was depleted from a slide and incubated with pepsin solution for $20 \mathrm{~min}$ at $37^{\circ} \mathrm{C}$. Tissue sections were covered with $3 \% \mathrm{H}_{2} \mathrm{O}_{2}$ to block endogenous peroxidase for 10 min at RT (room temperature). Slides were incubated with anti-EGFR at 1:50 (NICHIREI Bioscience) for $60 \mathrm{~min}$ at RT. After washing TBS (Tris-Buffered saline), the slides were incubated with a secondary antibody (EnVision $^{\mathrm{TM}}$ : DAKO JAPAN) for $30 \mathrm{~min}$ at RT. Tissue staining was visualized using a DAB (3, 3'-[[diaminobenzidine]]) substrate chromogen solution.

IHC staining for phosphorylated proteins was conducted as follows: After the same preprocessing of the
anti-EGFR antibody as described above, tissue sections were blocked with protein block serum free to prevent nonspecific reactions for $10 \mathrm{~min}$ at $\mathrm{RT}$. Slides were incubated with the primary antibody [pEGFR(1045) at 1:50, Cell Signaling Technology], [pERK (Thr202/ Tyr204) Rabbit mAb at 1:300, Cell Signaling] Technology overnight at $4^{\circ} \mathrm{C}$. The slides were then incubated with the secondary antibody (DAKO JAPAN) for 15 min at RT. Next, the slides were incubated with tyramide for $15 \mathrm{~min}$. Tissue staining was visualized using a DAB substrate chromogen solution. The slides were then counterstained with hematoxylin, dehydrated, and mounted.

\section{Results}

\section{Characterization of colorectal cancer cell lines}

Total EGFR protein expression was detected in HT29, JHCOLO-YI, JHSK-rec, HCT116, and TT1TKB by Western blot analysis. Three cell lines (HT29, HCT116, and TT1TKB) exhibited a relatively high level of protein expression, while one (JHCOLOYI) was intermediate, and 2 (CW2 and JHSK-rec) were very weak or showed no expression (Figure 1A). Sequencing analysis revealed that HT29, JHCOLO-YI, and CW2 were KRAS wildtype, whereas JHSK-rec, HCT116, and TT1TKB were KRAS mutant-type; JHCOLO-YI, JHSK-rec, HCT116, and TT1TKB were $B R A F$ wild-type, whereas HT29 was $B R A F$ mutant-type (Figure 1B) (Table 1).

\section{Growth suppression assay with cetuximab}

To determine the effects of cetuximab on cell growth in vitro, we performed a growth inhibitory assay with 6 CRC cell lines. A conventional chemosensitivity test using 5-FU or cetuximab was performed to identify if a growth inhibitory effect could be seen in a dose-dependent manner. As predicted, 5-FU exhibited a growth suppression curve with a dose-dependent manner in 4 out of 6 cell lines, whereas growth was not inhibited by cetuximab in any of the 6 cell lines (Figure 2).

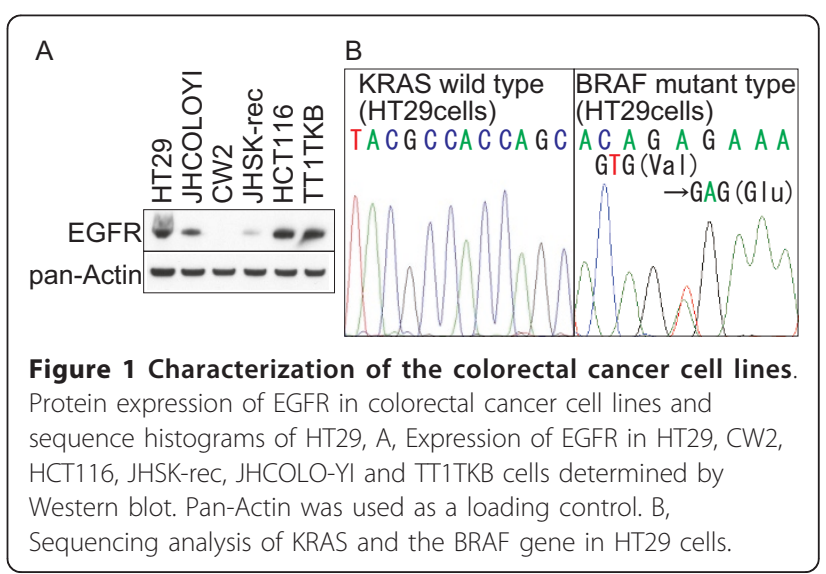


Table 1 Gene mutation status and EGFR expression of colon cancer cell lines

\begin{tabular}{lccc}
\hline Cell lines & EGFR & KRAS & BRAF \\
\hline HT29 & +++ & $w t$ & $\mathrm{mt}$ \\
JHCOLO-YI & ++ & $w t$ & $w t$ \\
CW2 & - & $w t$ & $w t$ \\
JHSK-rec & ++ & $\mathrm{mt}$ & $w t$ \\
HCT116 & ++ & $\mathrm{mt}$ & $w t$ \\
TT1TKB & ++ & $\mathrm{mt}$ & wt \\
\hline
\end{tabular}

$\mathrm{Wt}$, wild type; $\mathrm{mt}$, mutant type; +++, very strong positive; ++, strong positive; + , positive; -, negative. Level of EGFR is determined by Western blotting

\section{Signal transduction induced by growth factors and} cetuximab

All growth factors used in the present study activated EGFR/MAPK signaling (Figure 3 ) and TGF- $\alpha$ and IGF induced phosphorylation of downstream signaling more quickly than EGF did. While EGF induced sustained phosphorylation, a cocktail of TGF- $\alpha$ and IGF induced either exponential or temporal phosphorylation. We next observed for how cetuximab alters the phosphorylation level and whether it induces dephosphorylation in the presence of the above growth factors. As expected, the level of growth factor induced phosphorelation is decreased in the presence of cetuximab. Since all three growth factors have an affinity for EGFR, it makes sense that cetuximab reduces the phosphorylation levels that they induce; however, cetuximab does not seem to inhibit any of the phosphorylation levels completely. Interestingly, cetuximab itself exhibited the induction of phosphorylation in the absence of growth factors at multiple levels of the EGFR pathway.

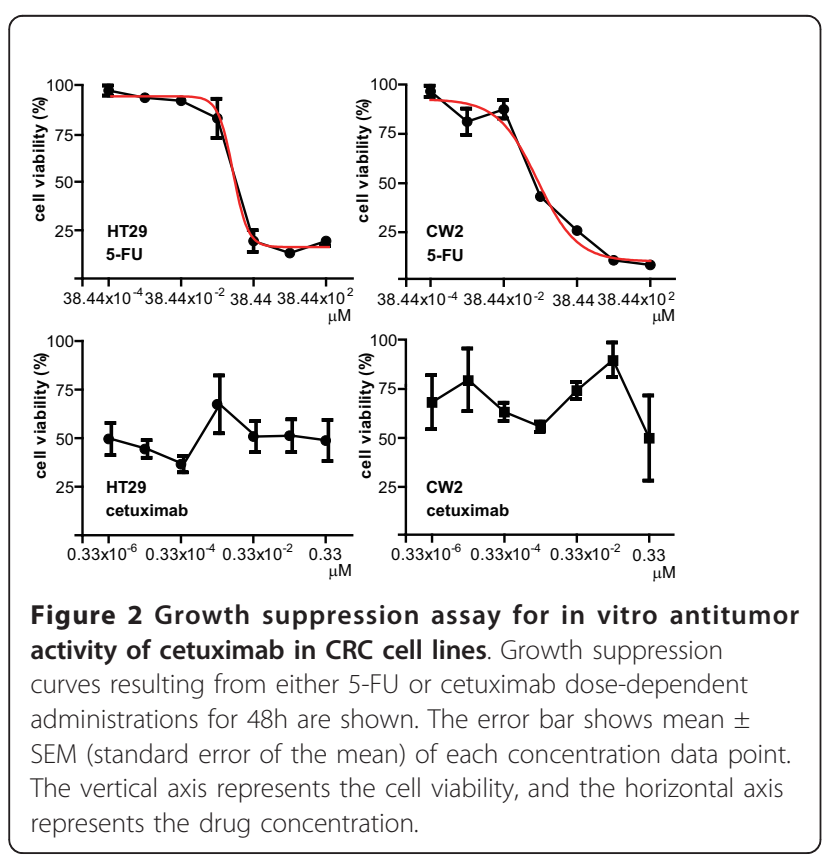

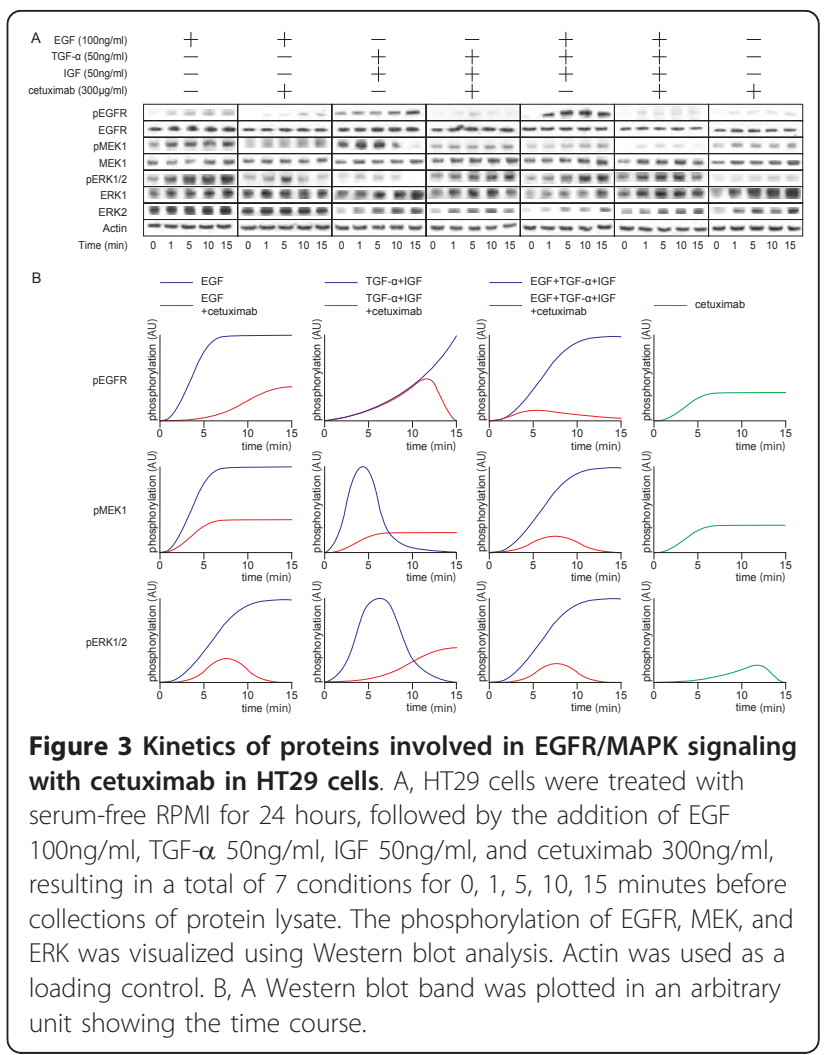

Inhibition of tumor growth in the mouse xenograft

Treatment with 6 injection of cetuximab for 26 days resulted in a significant suppression of tumor growth (P $<0.05$ ) (Figure 4). To evaluate whether there were any differences in the EGFR/MAPK signaling between cetuximab treatment (+) and (-) in vivo, we measured the total EGFR, phosphor-EGFR, and phosphor-ERK staining in the xenograft tumor sections. There were no visible differences between cetuximab (+) and cetuximab (-) tumors in terms of cell/tissue structure, and no necrotic or hyperinflammatory features were found. Both EGFR and phosphor-EGFR were stained in the cell membranes although the degree of staining was not homogeneous within the tumor. Phosphor-ERK nuclear staining was seen in substantial parts of the tumors. Interestingly, phosphor-ERK positive cells were occasionally found next to or in the negative cells (Figure 5).

\section{Discussion}

Expression of the EGFR protein was supposed to be the key molecule for cetuximab therapy indication. However, recent studies have indicated that EGFR IHC does not predict the need for cetuximab treatment. Subsequently analysis of the KRAS mutation has been widely accepted as giving a better prognostic value than EGFR $[7,8,19]$. In fact, our examination revealed that EGFR expression did not correlate with KRAS and BRAF 


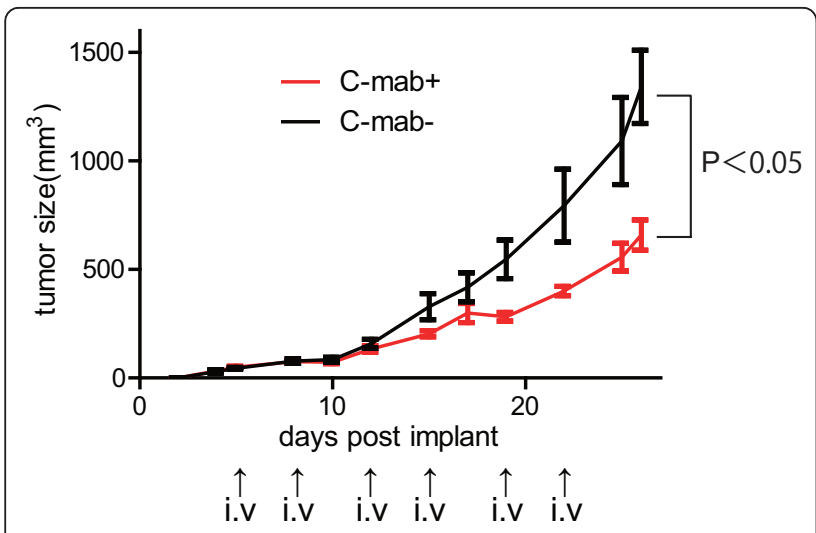

Figure 4 In vivo tumor suppression by cetuximab. Growth suppression of the HT29 mouse xenograft is present. HT29 xenografts were either untreated or treated with $200 \mu \mathrm{g}$ of cetuximab per mouse twice a week. The tumor volume was measured every 2-3 days, and all mice were sacrificed after 26 days of treatment. Error bars represent the standard error of the mean. We compared the tumor volume of treated and untreated xenografts using the student t-test $(p<0.05)$.

status. HT29 had the KRAS wild-type with BRAF mutation, and no $B R A F$ mutations were found in other cell lines with the mutated KRAS genotype. This is in agreement with previously published observations by other authors that mutations in KRAS and BRAF are mutually exclusive [20,21]. We chose HT29, which has the conventional criteria for cetuximab therapy (i.e. EGFR-positive and KRAS wild-type) to see how EGFR signal transduction was altered by the signal intervention with cetuximab.

In addition, we used a growth inhibitory assay to examine the cytotoxicity of cetuximab to clarify if a cellbased assay can be used in therapeutic decision making.
Surprisingly, cetuximab did not suppress cell growth in vitro in any of the 6 cell lines, including HT29. A possible explanation for this is that antibody-class anticancer agents including cetuximab do not seem to be as cytotoxic as other conventional classes of anticancer drugs, which may require a host-dependent cytotoxic mechanism (i.e., immune system) [22]. Hence, the conventional growth suppression assay may not work efficiently with the antibody class drugs, at least cetuximab.

HT29 is an EGFR-positive, KRAS wild-type and BRAF mutant-type cell line. In practice, it is considered that EGFR-positive as well as KRAS wild-type colorectal cancer patients have satisfied criteria for cetuximab therapy. It has also been speculated that colorectal cancer patients with the $B R A F$ mutant type may be considered to be a minor 'non-effective' group for cetuximab therapy as determined by clinical trials [6-8]. However, when it comes to individual patients, the grouping may not always be fully indicative. In fact, "the effective rate of KRAS mutant" and "non-response rate with the KRAS wild type" are $6.7 \%$ and $64.2 \%$, respectively [7]. Although these rates cannot be ignored they may not be of concern in practice because the gene status is currently almost the exclusive recommended criterion. In addition to showing the molecular mechanisms of tumor growth suppression by cetuximab, our results provide an example showing that it is worthwhile to consider not only the gene status but also the signaling pathway involved in the therapy.

All growth factors used in the present study activated EGFR/MAPK signaling and phosphorylation of downstream signaling was induced more quickly by TGF- $\alpha$ and IGF than EGF. This suggests that there is a crosstalk between EGFR/MAPK signaling and growth factor induced signaling other than EGF. While EGF induced

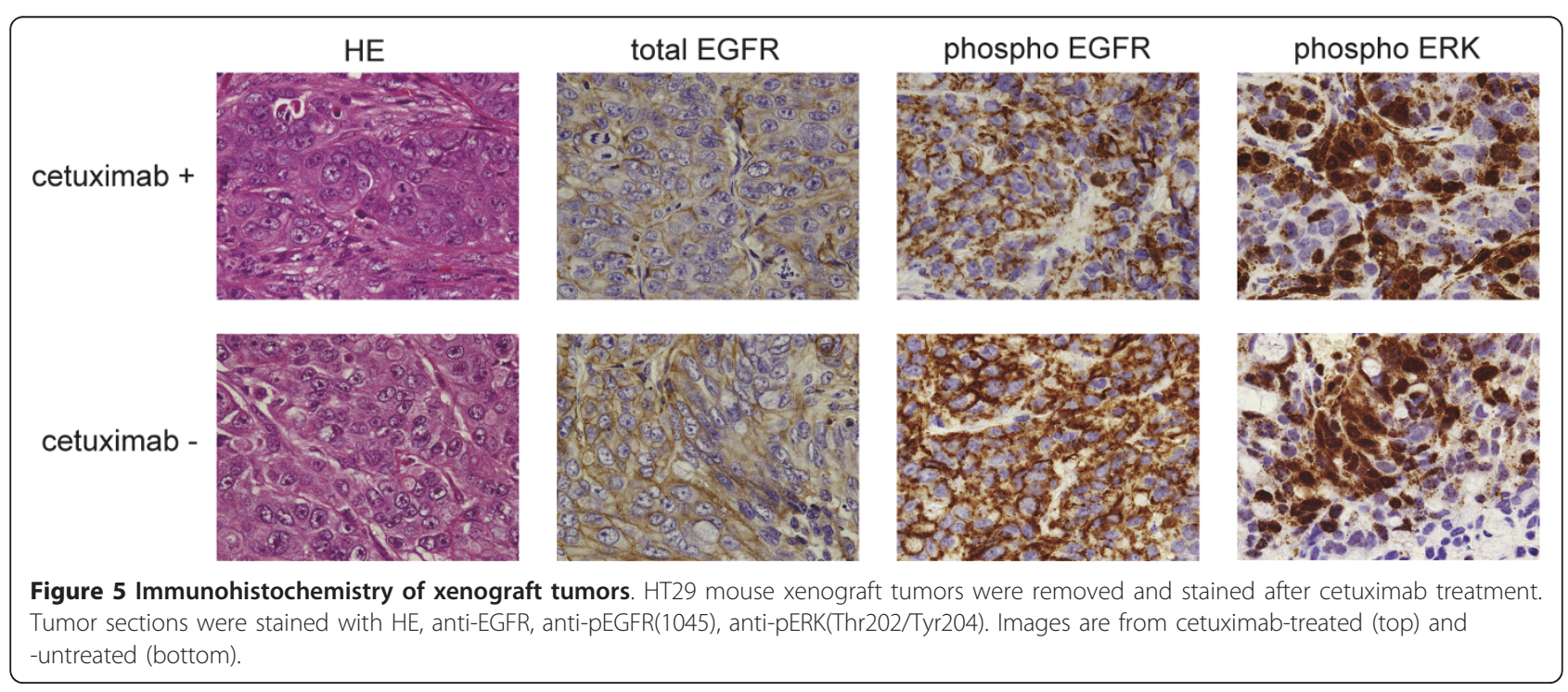


sustained phosphorylation, a cocktail of TGF- $\alpha$ and IGF induced either exponential or temporal phosphorylation, suggesting that there is a negative-feedback circuit, which regulates the level of phosphorylation at the MEK/ERK level. Interestingly, cetuximab itself exhibited the induction of phosphorylation in the absence of growth factors at multiple levels of the EGFR pathway (Figure 3). In fact, it has been reported that cetuximab itself induced phosphorylation of EGFR at several tyrosine phosphorylation sites as a result of receptor dimerization and activation of the receptor tyrosine kinase [23-25]. On the other hand, direct occlusion of the ligand-binding site is the primary mechanism of inhibition by cetuximab $[3,26]$. Moreover, cetuximab-EGFR complexes are not removed from the plasma membrane, in contrast to the rapid receptor turnover induced by EGF alone $[23,27,28]$. The ligand-EGFR complex is rapidly internalized and then either recycled back to the cell surface or proteolytically degraded. The internalized EGFR interacts with various signaling proteins that are important for sustained activation of the major signaling pathways mediated by ERK $[23,27,28]$. Taken together, the previous reports and our present results indicate that cetuximab leads to receptor dimerization that results in EGFR signal activation to some degree; but the dimerization is a less substantial signal inhibitor than the internalization of the ligand-EGFR complex (Figure 6).

Other Investigators have hypothesized the mechanisms of action of cetuximab as follows: (i) Inhibits internalization of EGFR resulting in a lack of downstream phosphorylation [23]; (ii) Induces apoptosis by starving growth signals [3,23]; (iii) causes an immunological response by provoking ADCC [22]; and (iv) fills up the EGF binding site preventing EGF from binding to its receptor [3].

To measure the result of such mechanisms of action, reflecting the drug's potency, we used a widely available method to measure cell growth using a water-soluble tetrasolium salt, WST-8, a substrate of formazan. This presumably reflects the number of cells in a microplate well (i.e. CCK-8 assay). Hence, the assay system reactions are maintained as long as cells generate energy from biochemical reactions that are present such as glycolysis even if their growth is somewhat suppressed. In the present study, we found no evidence for clear cetuximab dose-dependent growth suppression with the assay (Figure 2) in contrast to other cytotoxic drugs such as 5-FU, which showed clear logistic growth suppression curves. However, we found visible suppression of protein phosphorylation in the EGFR signal transduction pathways suggesting that cetuximab affects the signal transduction in the surviving cells that seem to be unaffected at the cellular level. This may imply that it is

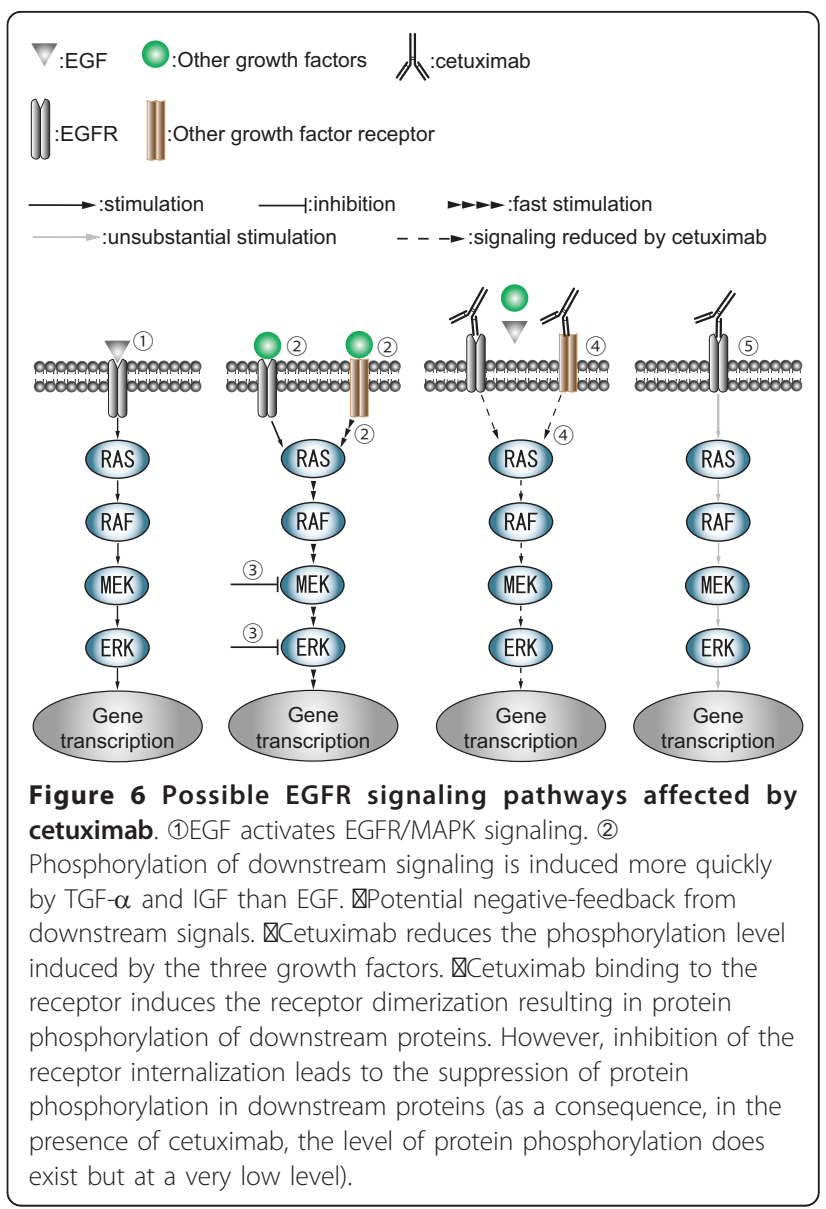

difficult to see the growth suppression from cetuximab alone in the short term, for example when using a $48 \mathrm{~h}$ cytotoxic assay. Based on previous reports and our present findings, it has been speculated that cetuximab may have a minimal direct cytotoxic effect by itself although an inhibitory effect of cetuximab on signal transduction in protein phosphorylation can be seen. The discrepancy between the growth suppression assay and the in vivo growth suppression effect of cetuximab may suggest that rapid growth suppression by cetuximab requires an extracellular effect.

The result of histological heterogeneous staining suggests that phosphorylation of the EGFR pathway components do not occur in a synchronized manner within the tumor. The fact that phosphor-EGFR positive cells are present in the tumor that has responded to cetuximab, also implies that cells which have acquired cetuximab may have selectively survived in response to the treatment.

\section{Conclusions}

Although KRAS gene mutation status is clinically useful for therapeutic decision making, the molecular effect of 
targeted molecules and the downstream pathways have not yet been fully elucidated. In the present study, we assessed the effect of cetuximab in tumor growth at 3 different levels including the protein and, cell levels, and in vivo, with gene status and EGFR expression information. At the protein level, EGFR signaling was effectively inhibited as a consequence of cetuximab exposure, whereas growth suppression assay did not show a significant effect probably due to the lack of direct cytotoxicity. However, CRC cell line xenografts followed by cetuximab administration to nude mice exhibited significant growth suppression. The phosphorylation status of the cetuximab target pathway did not visibly differ between cetuximab-treated and -untreated tumors removed from the mouse. These results suggest that the antitumor effect of cetuximab cannot be predicted by conventional growth suppression assay alone. Examination of the phosphorylation level seems to be informative, but the level does not synchronize in tumor cells probably due to the level change occurring in minutes order in a heterogeneous cell population. Although the set of assays is labor intensive and time consuming, it may compensate for the missing part in the current decision making process for selecting cetuximab therapy, leading to the establishment of new criteria for individual patients.

\section{List of abbreviations used}

CHAPS: 3-[(3-cholamidopropyl)dimethylammonio]-1-propanesulfonate; CRC: colorectal cancer 3, DAB: 3, 3'-[[diaminobenzidine]]; DMEM: dubecco's modified eagle medium; DMEM/F12: dubecco's modified eagle medium nutrient mixture F-12; DNA: deoxyribonucleic acid; DTT: dithiothreitol; EGF: epidermal growth factor; EGFR: epidermal growth factor receptor; ERK: extracellular signal-regulated kinase; FBS: fetal bovine serum; HE: Hematoxylin and eosin; IGF: insulin-like growth factor; IHC: Immunohistochemical; MAPK: mitgen-activated protein kinase; MEK: MAPK ERK kinase; PBS: Phosphate Buffered Saline; PCR: polymelase chain reaction; RPMI: Roswell Park Memorial Institute medium; RT: room temperature; TBS: tris-buffered saline; TGF-a: transforming growth factor-a

\section{Acknowledgements}

Authors thank Mr. Noriyuki Yamada. This work was partially supported by Grant-in-Aid for Scientific Research (C), (KAKENHI; Satoshi Nishizuka)

\section{Authors' contributions}

TM, SSN and GW conceived the study. TM, SSN, TI and GW were responsible for the experimental design. TM, SSN, KI, and MI performed experiments and helped in the analysis and interpretation. TM and SSN prepared the manuscript. All authors read and approved the final version of the manuscript.

\section{Competing interests}

The authors declare that they have no competing interests.

Received: 1 November 2010 Accepted: 10 May 2011

Published: 10 May 2011

\section{References}

1. Herbst RS: Review of epidermal growth factor receptor biology. Int J Radiat Oncol Biol Phys 2004, 59(2 Suppl):21-6.
2. Schneider MR, Wolf E: The epidermal growth factor receptor ligands at a glance. J Cell Physiol 2009, 218(3):460-6.

3. Schmitz KR, Ferguson KM: Interaction of antibodies with ErbB receptor extracellular regions. Exp Cell Res 2009, 315(4):659-70.

4. Goldstein NI, et al: Biological efficacy of a chimeric antibody to the epidermal growth factor receptor in a human tumor xenograft model. Clin Cancer Res 1995, 1(11):1311-8.

5. Blick SK, Scott LJ: Cetuximab: a review of its use in squamous cell carcinoma of the head and neck and metastatic colorectal cancer. Drugs 2007, 67(17):2585-607.

6. Van Cutsem E, et al: Cetuximab and chemotherapy as initial treatment for metastatic colorectal cancer. N Engl J Med 2009, 360(14):1408-17.

7. Roock De W, et al: Effects of KRAS, BRAF, NRAS, and PIK3CA mutations on the efficacy of cetuximab plus chemotherapy in chemotherapyrefractory metastatic colorectal cancer: a retrospective consortium analysis. Lancet Oncol 2010, 11(8):753-62.

8. Laurent-Puig P, et al: Analysis of PTEN, BRAF, and EGFR status in determining benefit from cetuximab therapy in wild-type KRAS metastatic colon cancer. J Clin Oncol 2009, 27(35):5924-30.

9. Tie J, et al: Optimizing targeted therapeutic development: Analysis of a colorectal cancer patient population with the BRAFV600E mutation. Int J Cancer 2010

10. Tie J, et al: Optimizing targeted therapeutic development: analysis of a colorectal cancer patient population with the BRAF (V600E) mutation. Int J Cancer 2011, 128(9):2075-84.

11. Nakanishi $Y$, et al: Loss of runt-related transcription factor 3 expression leads hepatocellular carcinoma cells to escape apoptosis. BMC Cancer 2011, 11:3.

12. Levin $V A$, et al: Different changes in protein and phosphoprotein levels result from serum starvation of high-grade glioma and adenocarcinoma cell lines. J Proteome Res 2010, 9(1):179-91.

13. Keese $\mathrm{M}$, et al: Imaging epidermal growth factor receptor phosphorylation in human colorectal cancer cells and human tissues. $J$ Biol Chem 2005, 280(30):27826-31.

14. Liu HS, et al: An unusual function of RON receptor tyrosine kinase as a transcriptional regulator in cooperation with EGFR in human cancer cells. Carcinogenesis 2010, 31(8):1456-64.

15. Jung YD, et al: EGCG, a major component of green tea, inhibits tumour growth by inhibiting VEGF induction in human colon carcinoma cells. $\mathrm{Br}$ J Cancer 2001, 84(6):844-50.

16. Jung YD, et al: Extracellular signal-regulated kinase activation is required for up-regulation of vascular endothelial growth factor by serum starvation in human colon carcinoma cells. Cancer Res 1999, 59(19):4804-7.

17. Mattingly RR: Mitogen-activated protein kinase signaling in drug-resistant neuroblastoma cells. Methods Mol Biol 2003, 218:71-83.

18. Nishizuka S, et al: Proteomic profiling of the $\mathrm{NCl}-60$ cancer cell lines using new high-density reverse-phase lysate microarrays. Proc Natl Acad Sci USA 2003, 100(24):14229-34.

19. Atkins $D$, et al: Immunohistochemical detection of EGFR in paraffinembedded tumor tissues: variation in staining intensity due to choice of fixative and storage time of tissue sections. J Histochem Cytochem 2004, 52(7):893-901.

20. Licar A, Cerkovnik P, Novakovic S: Distribution of some activating KRAS and BRAF mutations in Slovene patients with colorectal cancer. Med Oncol 2010.

21. Rajagopalan $\mathrm{H}$, et al: Tumorigenesis: RAF/RAS oncogenes and mismatchrepair status. Nature 2002, 418(6901):934.

22. Hsu YF, et al: Complement activation mediates cetuximab inhibition of non-small cell lung cancer tumor growth in vivo. Mol Cancer 2010, 9:139.

23. Yoshida $T$, et al: Matuzumab and cetuximab activate the epidermal growth factor receptor but fail to trigger downstream signaling by Akt or Erk. Int J Cancer 2008, 122(7):1530-8.

24. Fan Z, et al: Antibody-induced epidermal growth factor receptor dimerization mediates inhibition of autocrine proliferation of A431 squamous carcinoma cells. J Biol Chem 1994, 269(44):27595-602.

25. Li S, et al: Structural basis for inhibition of the epidermal growth factor receptor by cetuximab. Cancer Cell 2005, 7(4):301-11.

26. Sorkin A, Von Zastrow M: Signal transduction and endocytosis: close encounters of many kinds. Nat Rev Mol Cell Biol 2002, 3(8):600-14. 
27. Sorkin A: Internalization of the epidermal growth factor receptor: role in signalling. Biochem Soc Trans 2001, 29(Pt 4):480-4.

28. Suzuki Y, et al: Analysis of genetic alterations in renal cell carcinoma using the polymerase chain reaction. Virchows Arch 1994, 424(5):453-7.

doi:10.1186/1756-0500-4-140

Cite this article as: Matsuo et al: Analysis of the anti-tumor effect of cetuximab using protein kinetics and mouse xenograft models. BMC Research Notes 2011 4:140.

Submit your next manuscript to BioMed Central and take full advantage of:

- Convenient online submission

- Thorough peer review

- No space constraints or color figure charges

- Immediate publication on acceptance

- Inclusion in PubMed, CAS, Scopus and Google Scholar

- Research which is freely available for redistribution

Submit your manuscript at www.biomedcentral.com/submit
() Biomed Central 